\title{
Identification of spatial co-expression patterns and intra-tissue heterogeneity in spatially resolved transcriptomics by region-specific denoising
}

\section{Linhua Wang}

Baylor College of Medicine https://orcid.org/0000-0002-6717-860X

Zhandong Liu ( $\square$ zhandong.liu@bcm.edu )

Baylor College of Medicine https://orcid.org/0000-0002-7608-0831

\section{Article}

Keywords: Spatial transcriptomics, cell spatial organization, gene-expression

Posted Date: November 8th, 2021

DOl: https://doi.org/10.21203/rs.3.rs-647777/v1

License: (c) (1) This work is licensed under a Creative Commons Attribution 4.0 International License.

Read Full License

Additional Declarations: There is NO Competing Interest.

Version of Record: A version of this preprint was published at Nature Communications on November 14th, 2022. See the published version at https://doi.org/10.1038/s41467-022-34567-0. 
1 Identification of spatial co-expression patterns and intra-tissue heterogeneity in 2 spatially resolved transcriptomics by region-specific denoising

3 Linhua Wang ${ }^{1}$, Zhandong Liu ${ }^{2,3^{*}}$

4 1. Graduate Program in Quantitative and Computational Biosciences, Baylor College of Medicine, $5 \quad$ Houston, USA

6 2. Jan and Dan Duncan Neurological Research Institute at Texas Children's Hospital, Houston, USA

7 3. Department of Pediatrics, Baylor College of Medicine, Houston, USA

$8 \quad$ * Corresponding author: zhandonl@bcm.edu 


\section{Abstract}

We are pleased to introduce a first-of-its-kind tool that combines in-silico region detection and missing value estimation for spatially resolved transcriptomics. Spatial transcriptomics by $10 \mathrm{X}$ Visium (ST) is a new technology used to dissect gene and cell spatial organization. Analyzing this new type of data has two main challenges: automatically annotating the major tissue regions and excessive zero values of gene expression due to high dropout rates. We developed a computational tool-MIST_-that addresses both challenges by automatically identifying tissue regions and estimating missing gene-expression values for each detected region. We validated MIST detected regions across multiple datasets using manual annotation on the histological staining images as references. We also demonstrated that MIST can accurately recover ST'S missing values through hold-out experiments. Furthermore, we showed that MIST could identify intra-tissue heterogeneity and recover spatial gene-gene co-expression signals. We therefore strongly encourage using MIST before downstream ST analysis because it provides unbiased region annotations and enables accurately denoised spatial gene-expression profiles.

\section{Introduction}

To understand the biological mechanisms underlying diseases, it is essential to delineate cell and gene spatial organizations; the scientific community has therefore invested significant time and effort in detecting positional gene-expression ${ }^{1,2}$. Of all the spatial gene-expression profiling techniques that have been developed, 10X Visium Spatial Transcriptomics (ST) gained its most popularity due to its whole-genome scalability and cost-efficiency ${ }^{2,3}$. So far, ST has been used to study many tissues' spatial gene-expression, including breast cancer, prostate tumors, melanoma tumors, human and mouse brains, and more ${ }^{3-8}$.

While the location of every sequenced tissue domain (spot) in ST is assigned, regions of the tissue are not directly provided. Routinely, researchers circled out anatomical regions by looking at the histological staining image that is aligned with ST. However, in many cases, pathological regions are not detectable through human eyes. Moreover, assigning each spot to a region is

63 and unbiasedly extract regions within ST tissues. 
prevents accurate co-expression calculation, cluster detection, and other downstream analyses. Therefore, accurately estimating dropped-out expression values in ST data is vital to rescuing such signals and facilitating more accurate downstream analyses.

72 While we lack computational methods specifically designed to estimate missing values in ST, 73 plenty of such methods have been developed for single-cell RNA sequencing (scRNA-seq) 74 data ${ }^{9-14}$. Many of these methods - like MAGIC ${ }^{9}$, PRIME $^{10}$ and knnSmoothing ${ }^{11}$-smooth the 75 missing gene expression values by taking the weighted average of the gene expression values 76 from cells with similar molecular profiles. None of these methods, however, interpret ST's spatial 77 connectivity information to determine the functionally and physically similar neighbors to learn 78 from. Other kinds of methods-like Mclmpute ${ }^{12}$ and ALRA ${ }^{14}$, estimate the missing values by 79 approximating a low rank of the gene expression matrix, assuming a relative number of cell 80 types within the tissue. However, they are performed at the entire tissue and lack regional 81 specificity that could allow us to rescue regional heterogeneity.

83 We realized that these two challenges: (1) difficulty automatically annotating tissue regions and 84 (2) excessive zero gene expression values due to technical dropouts are not independent of each other. On the contrary, solving the first challenge could facilitate region-specific denoising.

87 An intuitive way of denoising ST using spatial information is to use spatially adjacent neighbors to estimate missing values. However, using adjacent neighbors introduces errors regarding spots on functionally different tissue regions' boundaries. In such cases, detecting the boundaries is vital to avoiding spatial-information misusage when denoising ST data and allows ST to be grouped into functionally similar regions. Denoising within such functional and spatial

92 regions, therefore, avoids errors at tissue boundaries.

We hypothesize that the number of cell types within a functional region in the tissue is limited, leading us to believe that a more accurate denoising result will be achieved by region-specific

96 denoising through low-rank matrix completion. Therefore, we introduce our new tool - Missing-

97 value Imputation for Spatially resolved Transcriptomics (MIST) that addresses the in-silico

98 detection and region-specific denoising tasks in a two-step pipeline.

\section{Results}

101 The MIST Algorithm 
MIST provides a two-step tool to address the challenges in ST's analyses including automatically detecting ST's functional regions and denoising ST data at a region-specific schema (Fig. 1).

In the first step, MIST automatically detects functional regions in the sample of interest. MIST embeds ST as a 2D graph where every spot will be presented as a node. Every pair of adjacent

To simulate the region boundaries, MIST filtered out low-weight edges with a threshold and extract the connected components within the remaining graph. To avoid bias in selecting the filtering threshold, MIST searches for the optimal value by assessing different thresholds' accuracy in recovering the hold-out values of a set of highly expressed genes. nodes will have an edge connecting each other with a weight defined by the molecular similarity between the pair of nodes.

In the second step, MIST estimates the missing values in each detected region by averaging the outcomes from multiple runs of a low-rank approximation algorithm ${ }^{12}$. Under the assumption that each detected region should have a small number of cell types, we expect the denoised regionspecific expression matrix to have a low rank. To achieve this goal, we used a low-rank matrix completion approach that estimates the missing values by minimizing the singular values of the denoised matrix.

To increase the reliability of the estimated values and avoid an extremely singular geneexpression matrix, before denoising, MIST adds contrast to each region by augmenting the region with random spots from other regions. To remove the bias from randomly selected spots and stabilize the estimated values, MIST runs multiple repeats of low-rank approximation on the augmented regions with different sets of augmented spots and takes the average of the outcomes as the estimated values for region-specific spots.

\section{MIST detected functional tissue regions that agree with manual annotations}

132 two examples including a melanoma sample and a mouse brain sample (Fig. 2a, b). We

133 showed that under different filtering thresholds, different shapes of regions were detected.

134 However, regions using the threshold automatically selected by MIST mostly resembles the 135 manual annotation provided by human experts. 
137 While higher filtering thresholds on the edges will find strongly correlated spots within regions,

138 they also lead to a significant number of isolated spots with no connected edges (Fig. 2c). But, 139 while small thresholds will include most spots, they will lose the specificity that allows us to 140 accurately detect tissue regions (Fig. 2c). Balancing the proportion of non-isolated spots and 141 region-detection accuracy is, therefore, crucial to successful region detection and region142 specific denoising.

To demonstrate that MIST strikes this balance automatically and accurately, we used Adjusted

145 Rand Index (ARI) to evaluate the consistency between MIST-detected regions and the human 146 experts' annotations. An ARI of zero shows the concordance of two random clustering results 147 while a score of one indicates a perfect match of two clustering results. Using the mouse wild148 type brain sample, we validated that the threshold that MIST automatically selected found a 149 balance point that maximizes the ARI while minimizing the proportion of isolated spots (Fig. 2c). 150 Using the selected threshold, the mouse brain regions detected by MIST agreed with the anatomical regions (Fig. 2b) with an ARI value of 0.64 .

To confirm the biological relevance of MIST-detected regions in the melanoma sample, we looked at the gene profiles in these regions. Specifically, we identified significantly activated genes in the tumor region relative to the lymphoid region and vice versa using the Wilcoxon rank-sum test. By selecting genes with a log2 fold-change greater than 0.58 and an adjusted Pvalue less than $10^{-5}$, we found 143 and 49 marker genes for the tumor and lymphoid regions, respectively (Fig. 2d). We observed that some well-known melanoma marker genes, including $M L A N A^{15}, M_{C A M}{ }^{16}, S P P 1^{17}$, and $H S P 90 A A 1^{18}$, topped the list of the melanoma-activated genes that we identified, which demonstrates our detected region's accuracy. We then performed gene set enrichment analysis on the significantly activated genes and observed that the immune

162 response gene ontology term was significantly enriched (adjusted P-value $=9 \times 10^{-8}$, Fig. 2e)

163 for the detected lymphoid region. Taken together, the region automatically detected from the

164 Melanoma ST reflected the hidden functional regions within the tissue.

\section{MIST accurately recovers hold-out values across multiple data sets}

167 To assess MIST's accuracy when estimating missing values, we performed 5-fold random holdout experiments in which we withheld a random set of the observed non-zero values and used these as ground truth to evaluate the models' performances. By withholding some of the 
170 observed values, we simulated cases in which non-zero expression values have dropped out.

171 To consider the heterogeneity of datasets that might lead to biased performance, we tested four

172 datasets from samples including a wild-type mouse brain sample, an AD mouse brain sample, a

173 melanoma tumor sample, and a prostate tumor sample that vary in the number of genes,

174 number of spots, and sparsity level (Table 1). For each dataset, we selected genes that are

175 expressed across at least half of all spots in the sample to generate hold-out test data sets. For

176 each gene, we partitioned the non-zero expression values into five non-overlapping sets. Then,

177 we iteratively held out one-fold of the values and assessed MIST and other methods' accuracy

178 in recovering the held-out gene expression values.

179

180 To demonstrate MIST's supremacy in missing value recovery, we compared MIST with state-of-

181 the-art scRNA-seq methods ${ }^{19}$, including MAGIC ${ }^{9}, \mathrm{knn}^{-s m o o t h i n g}{ }^{11}$, Mclmpute $^{12}$ and SAVER ${ }^{13}$,

182 and a baseline k-nearest neighbor method we constructed (spKNN) that estimates missing

183 values by averaging spatially adjacent neighbors.

To evaluate the accuracy of missing value estimation in hold-out experiments, we used two metrics including Rooted Mean Square Error (RMSE) and Pearson Correlation Coefficient (PCC) where RMSE represents the error and PCC shows the agreement between the ground truth and estimated values. Better imputation methods are expected to have lower RMSE and higher PCC scores.

We found that MIST consistently outperformed other methods across all datasets with higher PCC and lower RMSE scores during hold-out value evaluation (Fig. 3a, b). For the spots that are assigned to functional regions, MIST had an average RMSE improvement (decreasing values) of $12 \%$ (P-value $=3 \times 10^{-4}$ compared with Mclmpute, and $14 \%\left(P\right.$-value $\left.=10^{-14}\right)$ and $35 \%\left(P\right.$-value $\left.=7 \times 10^{-10}\right)$ improvement compared with the baseline spKNN algorithm.

196 Moreover, MIST's mean PCC is also $8 \%\left(P\right.$-value $\left.=9 \times 10^{-6}\right), 4 \%\left(P\right.$-value $\left.=2 \times 10^{-4}\right)$, and $16 \%$

197 (P-value $=6 \times 10^{-11}$ ) higher than Mclmpute, MAGIC and spKNN respectively. Knn-smoothing 198 and SAVER consistently performed substantially worse than the other four methods (Ext. Fig. $1993.1,3.2)$.

201 To investigate the impact of genes' sparsity on denoising accuracy, we further stratified the 202 performance assessment at a per-gene level grouped by the sparsity level (zero-value 203 proportion). While MAGIC and spKNN's estimation error monotonically increased with sparsity 
204 level, MIST's performance was not vulnerable to gene sparsity (Fig. 3c). While Mclmpute's

205 performance was also not influenced by gene sparsity, MIST has superior performance than

206 Mclmpute at every gene sparsity level (Fig. 3c).

207

208 To demonstrate that MIST recovers the gene-expression patterns, we then visualized and 209 showed that MIST can faithfully recover the gene-expression spatial pattern for gene GAPDH 210 after denoising using the Melanoma tissue sample (Fig. 3d). With the hold-out input, MIST

211 accurately estimated the original expression values by increasing Spearman's correlation

212 coefficient from 0.65 to 0.96 (Fig. 3e). When evaluating all genes across the four tested data

213 sets, after denoising, the median correlation had significantly improved from 0.64 to 0.88 (P-

214 value $\approx 0$, Fig 3f, Ext. Fig. 3.3, 3.4).

MIST discovered intra-cortex heterogeneity within an Alzheimer's Disease (AD) mouse

218 To demonstrate that MIST could improve the clustering results of ST data, we used Uniform Manifold Approximation and Projection (UMAP) ${ }^{20}$ to reduce the dimension of Mouse brain ST samples' gene expression data and visualize the clustering structures. First, we performed

221 UMAP on the original and denoised wild-type mouse brain sample. After denoising, MIST

222 enhanced the heterogeneity with most of the spots within the cortex, hippocampus, and

223 thalamus, forming individual clusters (Fig. 4a, Ext. Fig. 4.1).

While similar enhanced clustering patterns were also identified in the Mouse AD brain sample after denoising, in addition, we observed that the cortex region was separated into two individual clusters, something we did not detect using the WT mouse brain nor in the original Mouse AD data (Fig. 4b, Ext. Fig. 4.2). Further cortex analysis revealed a clear separation of the cortex region into two spatially separable parts (Fig. 4c, Ext. Fig. 4.3). The first cluster consisted of the cortical subplate, olfactory, entorhinal, ectorhinal, temporal association, and perirhinal areas.

231 The second cluster contained the auditory, primary somatosensory, posterior parietal

232 association, and retrosplenial areas. When these two clusters were mapped to the anatomical 233 reference, cluster 1 occupied the upper quadrant while cluster 2 occupied the lower quadrant 234 (Fig. 4d-f). Interestingly, such heterogeneity was only detected in the MIST-denoised AD cortex 235 but not the WT cortex. 
237 To understand the biological difference of these two clusters in AD progression, we further

238 performed differential gene analysis to extract AD activated genes for these two clusters,

239 respectively. By selecting upregulated genes in the AD sample with a fold change greater than

$24050 \%$ and adjusted P-value < 0.01, we identified 55 markers for cluster 1 and 41 markers for

241 cluster 2 (Ext. Fig. 4.4, 4.5). We found only 21 AD-activated genes, such as Clu, are shared for

242 these two clusters (Fig. 4d). 34 genes, such as Hap1, are AD-upregulated only in cluster 1 (Fig.

$2434 \mathrm{e}$ ) and 20 genes, such as Sez6, that are only AD-upregulated in cluster 2 (Fig. 4f). We showed

244 that the spatial clusters identified using MIST-denoised cortex data play different roles in AD

245 progression by activating different sets of genes, demonstrating that MIST allows the discovery

246 of biological heterogeneity from ST data.

\section{MIST recovers spatial gene-gene co-expression patterns}

Spatial gene-gene co-expression plays an important role in understanding gene interactions across 2D space. The dropouts within the ST data undermine the correlation analysis's power and cause inaccurate estimation of gene-gene spatial correlation, which is the fundamental element in many analyses such as weighted correlation network analysis (WGCNA) ${ }^{21}$.

To demonstrate that MIST recovers the spatial co-expression patterns, we examined two pairs of genes: Cldn11-Arhgef10 and Gfap-Aqp4.

The first pair of genes Cldn11-Arhgef10 showed a high spatial correlation score of 0.97 based on the reference Allen Brain Atlas ${ }^{22}$ (Fig. 5a). However, in the original ST data, the correlation score between Cldn11 and Arhgef10 is only 0.15 (P-value $=2 \times 10^{-3}$, Fig. 5b). After denoising by MIST, the correlation score was improved to 0.5 (P-value $=3.5 \times 10^{-29}$, Fig. $5 \mathrm{c}$ ). To visualize the gene expression patterns, we plotted the heatmap of log-scale expression values and

262 showed that Cldn11 and Arhgef10 have similar gene expression patterns only after MIST 263 denoising (Fig. 5d-g).

To show that MIST can recover co-expressed gene pairs that are not significantly correlated with the original data, we examined the second pair of genes: Gfap-Aqp4, which have a moderately good spatial correlation with a score of 0.74 in the reference Allen Brain Atlas database (Fig. 5h). Before denoising, we observed an insignificance correlation with a score of 0.08 (P-value $=0.56$, Fig. 5i). After denoising, we could recover a significant spatial correlation 
271 pattern improvement of Gfap-Aqp4 could also be observed only after denoising by MIST (Fig.

$2725 k-n)$.

273

274 By showing these two cases, we demonstrated that MIST could rescue the spatial correlation of 275 gene pairs whose co-expression patterns are either lessened or lost in the original ST data.

276 Given co-expression estimation is vital in many downstream analyses such as identifying gene

277 modules $^{21}$, we see the significance and importance of using MIST to denoise ST data before

278 carrying out such analyses.

279

\section{Conclusions}

281 In this study, we tackled two major problems encountered in ST data analyses - (1) in-silico 282 region detection and (2) missing value estimation. We developed a computational tool, MIST, 283 that solved both problems.

We solved the first challenge by combining the molecular similarity and spatial connectivity between spots and enabled detecting tissue regions automatically. Before our work, researchers align the histological images with ST coordinates to manually assign regions to every spot. This procedure is laborious and might be inaccurate in cases where pathological changes are not visually detectable through human eyes. With MIST, we could bypass manual annotation and allows unbiased region assignment to every spot. We proved that MIST could accurately detect regions by comparing MIST-detected regions with manually assigned regions

292 at the per-spot level. Addressing this challenge allows us to analyze ST data at a region-specific 293 level and improve the specificity for denoising.

We solved the second challenge by approximating a low rank of region-specific gene expression matrix through singular value decomposition. This is based on a simple yet

297 interpretable assumption that the number of cell types for every region is small, which has been

298 adopted by many other single-cell RNA-sequencing denoising methods such as Mclmpute ${ }^{12}$ and 299 ALRA $^{14}$. Compared with Mclmpute and ALRA, MIST utilized spatial information to define regions 300 and improved the denoising specificity.

302 To demonstrate that MIST's accuracy in estimating the missing values, we developed hold-out 303 experiments to simulate dropout events and assessed MIST's performance in the hold-out trials. 
As a result, MIST could accurately recover the hold-out values and outperformed the state-ofthe-art single-cell denoising methods and a baseline spatial-information-based approach.

While addressing these two major challenges, MIST allows researchers to drastically recover

308 biological signals that are missing in the original ST data. Such biological signals include spatial co-expression patterns, intra-region heterogeneity, and more.

We demonstrated that MIST could improve or recover spatial patterns of co-expressed genes

312 that are highly correlated in reference atlas but either poorly or insignificantly correlated in the 313 original ST. Since many downstream analyses such as WGCNA ${ }^{21}$ are based on co-expression estimation, denoising ST by MIST is rather essential before such analyses because it avoids false conclusions due to inaccurate co-expression estimation.

It is also of great significance to understand the heterogeneous response of different tissue regions to pathology, which will shed light on the treatment of diseases. Therefore, it is critical to recovering such heterogeneity that was lost in the original ST data.

321 We showed that MIST enables recovering the spatial heterogeneity within the mouse cortex

322 during $A D$ progression. By extracting the differentially expressed genes between AD and wild-

323 type mouse brain samples, we identified genes that are AD-upregulated only in specific regions

324 of the mouse cortex.

326 We therefore unequivocally recommend using MIST for ST analyses. Our sui generis

327 in-silico region detection enables analyzing the ST at a brand-new level that combines

328 anatomical connectivity and molecular similarity. It will be useful in many areas, including

329 identifying local subregions within tumors whose heterogeneity is hard to see through

330 pathological staining images. Second, MIST allows researchers to recover important biological

331 signals in downstream analyses, such as when identifying spatial gene-gene co-expression

332 patterns. While the original ST data provided by 10X Visium might hinder ST analyses'

333 accuracy, MIST will accurately rescue the missing values and drastically increase the signal-to334 noise ratio.

\section{Methods}

337 Data collection and preprocessing 
The ST data sets we used in this study include a 12-month wild-type mouse brain sample, a 12-

339 month Alzheimer's Disease (AD) mouse brain sample, a Melanoma tumor sample, and a

340 prostate tumor sample $5,6,8$ (Table 1). Every dataset has a raw mRNA count matrix form where

341 rows indicate spots and columns indicate genes.

343 To filter out low-quality genes that might otherwise introduce noise to the pipeline, we kept

344 genes with raw counts $>=3$ in more than 2 spots within each sample.

346 To account for the different library sizes of every spot due to variance in sequencing depth and

347 the number of cells, we normalized the raw mRNA count matrix using count per million ( $C P M=$

$348 \quad \frac{\text { Raw count } * 10^{6}}{\text { Library size }}$ ).

\section{In-silico region detection}

\section{Graph embedding}

352 Suppose the ST expression matrix has $M$ spots and $N$ genes, the spatial gene-expression

353 profile can be defined as $Y \in R_{*}^{M \times N}$, where $Y$ is the observed gene expression matrix, and $R_{*}$

354 denotes non-negative real matrices with $M$ rows and $N$ columns. The $M$ spots in an ST slide can

355 form a lattice graph, $G=\langle V, E\rangle$, where $\mathrm{V}$ is the node-set and $E$ is the edge-set. Every pair

356 of adjacent $(u, v)$ spots are connected with edge $E(u, v)$.

Weight calculation

359 To infer the weights for every connected edge $E(u, v)$, we calculated the Pearson correlation 360 coefficient between the gene expression profile of spot $u$ and $v$. To remove the noise in high361 dimensional gene expression data while keeping the major signals, we used Principal

362 Component Analysis (PCA) ${ }^{23}$ to reduce the dimension of the gene expression matrix and kept 363 the first $p$ principal components that could explain $80 \%$ of the variance. The weight for edge $E$ $364(u, v)$ is then inferred using the correlation score between the first $p$ principal components of 365 spot $u$ and $V$.

Edge removal and parameter selection

368 To simulate the boundaries between functionally dissimilar regions, we removed edges whose 369 weights are less than a threshold $\varepsilon$. 
371 To automatically select the threshold and avoid bias, we performed a hold-out experiment on a

372 set of genes that are expressed in at least $80 \%$ of all spots. We randomly held out $25 \%$ of the

373 gene expression values for every such gene and used them as ground truth to test MIST's

374 denoising error defined as $R M S E=\sqrt{\frac{\sum_{i=1}^{n}\left(X_{i}-X_{i}^{\prime}\right)^{2}}{n}}$, where $X$ is the hold-out non-zero values, $X^{\prime}$

375 represents the MIST denoised gene expression matrix using certain $\varepsilon$ and $n$ denotes the number

376 of non-zero hold-out values. To determine the optimal threshold, we did a grid search with

377 values ranged from 0.1 to 0.9 and selected the one with the minimal RMSE.

378

Region detection by extracting connected components

380 To simulate the functional regions within tissues, we used a depth-first search algorithm ${ }^{24}$ to

381 identify all the connected components in graph $G$ after edge removal. Each connected

382 component with greater than 5 spots is predicted as an independent tissue region.

384 Region-specific denoising through multiple runs of low-rank matrix completions

\section{Low-rank matrix completion}

386 Suppose the observed gene expression matrix for region $C$ is $Y_{C}$ where $Y_{C}$ is a sparse matrix

387 with $m$ rows (spots) and $n$ columns (genes). The task is to estimate $X_{C}$, which represents the 388 denoised gene expression matrix for region $C$. We adapted the low-rank-matrix completion 389 algorithm through singular value decomposition used by Mclmpute ${ }^{12}$.

391 Given the assumption that the number of cell types within a functional region is small, we expect $392 X_{C}$ to have a low rank. To achieve this goal, the task is turned into a low-rank matrix completion 393 problem by solving the following objective function:

$394 \min _{X_{C}}|| Y_{C}-A\left(X_{C}\right) \|^{2}+\lambda * \operatorname{rank}\left(X_{C}\right)$

396 The first term in equation (1) minimizes the error between the non-missing gene expression 397 values of $X_{C}$ and $Y_{C}$ with a projection function $A$ that returns values in $X_{C}$ at the indices of non398 missing values in $Y_{C}$. The second term in equation (1) minimizes the rank of the denoised gene 399 expression matrix. The objective function is a linear combination of these two terms regularized 400 a non-zero tuning parameter $\lambda$. Theoretically, a larger $\lambda$ will give us a lower-ranked denoised 401 gene expression matrix whose values on the non-missing indices might deviate from the ground 
truth. On the other hand, a small $\lambda$ will result in a relatively high-rank denoised matrix with a

403 lower error on the non-missing indices.

404

405 However, minimizing the rank of a matrix is non-convex. To transform it to a convex problem

406 with a globally optimal solution, we relaxed equation 1 as:

$407 \min _{X_{C}}\left|\left\|Y_{C}-\left.A\left(X_{C}\right)\right|^{2}+\lambda *\right\| X_{C} \|_{n u c}\right.$

408 , where we transformed the term 2 in equation 1 as a nuclear norm of $X_{C}$, which can be 409 calculated by summing up the singular values obtained through singular value decomposition.

410 Specifically, equation (2) can be further transformed as

$411 \min _{X_{C}}|| B-X_{C}\left\|^{2}+\lambda *\right\| X_{C} \|_{n u c}(3)$,

412 where $B_{k+1}=X_{k, C}+\frac{1}{\alpha} A^{T}\left(Y_{C}-A\left(X_{k, C}\right)\right)$. Using the inequality $\|B-X\|_{2} \geq\left\|s_{B}-s_{X}\right\|$, where $s_{X}$ 413 denotes the singular value vector for matrix $X$, equation (3) can be rewritten as

$414 \min _{X_{C}}|| s_{B}-s_{X_{C}}\left\|^{2}+\lambda *\right\| s_{X_{C}} \|_{1}(4)$.

415

416 Therefore, by taking the derivative of (4), the solution of $s_{X_{C}}$ is achieved by soft thresholding the 417 singular values of $s_{B}$ with a threshold equals to $\lambda / 2$.

419 To tune the parameter $\lambda$ that strikes the balance between low matrix singularity and low error on 420 non-missing indices, we find the maximal $\lambda$ that achieves a fixed low error $\left(10^{-12}\right)$ calculated by

421 the sum of the absolute difference between denoised and observed values on non-missing 422 indices.

423

424 Compared with the original solution provided by Mclmpute, MIST also forces the observed 425 values to be unchanged. By doing so, MIST guaranteed that it will only touch the missing values 426 and keep the observed non-zero expression values unharmed. Moreover, MIST's matrix 427 completion was implemented in a free and open-source language-Python, as compared to 428 MATLAB, which was used by Mclmpute.

Region augmentation

431 To avoid an extremely singular denoised gene expression matrix that might otherwise introduce 432 instability, we added contrast to every detected region before low-rank matrix completion.

433 Specifically, we augmented the region $C$ with $\mathrm{r}$ randomly selected spots from other regions 
434 where $r=\min \left(\frac{\operatorname{size}(\text { Other regions) }}{10}\right.$, size $\left.(C)\right)$. The low-rank matrix completion algorithm will be

435 performed on the augmented region $C^{a}$ with input $X^{a}$.

437 To include more diversity and reduce the randomness in region augmentation, we repeated the

438 previous step $\mathrm{T}$ times. For every spot $s \in C$, we take the average of denoised gene expression

439 values $Y^{a}(s)$ from outcomes of $\mathrm{T}$ repeats. $\mathrm{T}$ is set to be 10 as a default to avoid high

440 computational load while including as much signals as possible.

442 To demonstrate the essentiality of region augmentation, we tested MIST's performance in the 443 hold-out experiments using detected regions and augmented regions, respectively. We found 444 that region augmentation significantly improved MIST's denoising accuracy (RMSE P-value = $4451.6 \times 10^{-7}$, PCC P-value $=4.4 \times 10^{-7}$, Ext. Fig. 6).

\section{Quantifying region detection accuracy}

448 Adjusted rand index is used to quantify the agreement between the manual spot-level

449 annotation and MIST-predicted regions. A score of one means a perfect agreement between

450 two grouping results while zero score means the predicted regions are similar to the results from

451 random guessing. Adjusted rand index scores are calculated using the Python scikit-learn

452 package $^{26}$.

\section{Differential gene expression analysis}

455 Wilcoxon rank-sum test provided by the Python package Scipy ${ }^{25}$ was used to infer the 456 significant level of differentially expressed genes. Fold change of genes between condition and 457 control was calculated based on the difference of average gene expression within groups. To 458 get the significantly upregulated genes in the MIST-predicted melanoma region relatively to 459 lymphoid region, we selected genes with a fold change greater than $50 \%$ and adjusted $\mathrm{P}$-value 460 less than $10^{-5}$. To get the AD upregulated genes in the mouse cortex regions, we changed the 461 adjusted $\mathrm{P}$-value threshold to $10^{-2}$ because of smaller sample sizes.

\section{Gene set enrichment analysis}

464 The R package clusterProfiler ${ }^{27}$ was used to perform gene ontology enrichment analysis using 465 the list of differentially expressed genes in the melanoma sample. 
468 To improve the diversity in the hold-out experiments, we tested samples including a mouse wild469 type brain sample, a mouse AD brain sample, a melanoma tumor sample, and a prostate tumor 470 sample. To extract good-quality genes to simulate the dropout events, we removed genes that 471 were expressed in less than $50 \%$ of the spots.

472

473 To generate the hold-out data, we used a 5-fold cross-validation schema for the non-zero

474 values. Specially, we first randomly partitioned every gene's nonzero expression values into 5

475 groups. In each hold-out, we created missing values by setting one group to zero and performed 476 imputation based on the remaining values. The held-out values served as ground truth for 477 evaluating the imputation algorithms' accuracy.

\section{Evaluating hold-out experiments' performance}

480 To quantifying the accuracy in recovering the held-out values, we reported two metrics including 481 RMSE and PCC, where RMSE measures the estimation error and PCC measures the linear 482 correlation between the true expression values and the estimated values. RMSE is defined as

$483 R M S E=\sqrt{\frac{\sum_{i=1}^{n}\left(X_{i}-Y_{i}\right)^{2}}{n}}$ and PCC is defined as PCC $=\frac{\sum_{i=1}^{n}\left(X_{i}-\bar{X}\right)\left(Y_{i}-\bar{Y}\right)}{\sqrt{\sum_{i=1}^{n}\left(X_{i}-\bar{X}\right)^{2}} \sqrt{\sum_{i=1}^{n}\left(Y_{i}-\bar{Y}\right)^{2}}}$ where $Y$ is the hold-

484 out non-zero values, $X$ represents the MIST estimated values and $n$ denotes the number of 485 hold-out values.

487 To quantify the recovery of gene expression patterns after denoising the hold-out data, we used 488 Spearman's rank correlation test implemented by Scipy ${ }^{25}$ to assess the correlation and the corresponding significance level between the original ST and denoised gene expression values.

Co-expression analysis using Allen Brain Atlas as a reference

492 We obtained mouse brain (coronal section) regional expression values for gene Cldn11

493 (experiment: RP_070116_01_G04), Arhgef10 (experiment: RP_070116_01_B05), Gfap

494 (experiment: RP_Baylor_253913) and Aqp4 (experiment: RP_040324_01_F07) from Allen

495 Brain Atlas ${ }^{22}$ as references. Gene expression values provided by Allen Brain Atlas are at the $496 \log 2$ scale.

498 Correlation scores between gene pairs in both reference and ST data are represented by 499 Spearman's correlation coefficient calculated using the Python package Scipy ${ }^{25}$. 
Table 1. Data summary for hold-out experiments

\begin{tabular}{c|cccc} 
& MouseWT & MouseAD & Melanoma & Prostate \\
\hline \#Genes (preprocessed) & 10178 & 11241 & 4498 & 8073 \\
\#Genes (<50\% sparsity) & 3024 & 4081 & 952 & 1718 \\
\#Spots & 447 & 488 & 293 & 406 \\
Non-zero values (\%) & 22 & 26 & 15 & 21
\end{tabular}

502

\section{Source Code Availability}

504 The MIST algorithm is implemented in Python and is available at

505 https://github.com/linhuawang/MIST.git.

506 All the source code and raw data for this manuscript can be downloaded from

507 https://github.com/LiuzLab/MIST manuscript.git.

508

\section{References}

510 1. Marx, V. Method of the Year: spatially resolved transcriptomics. Nat. Methods 18, 9-14

511 (2021).

512 2. Asp, M., Bergenstråhle, J. \& Lundeberg, J. Spatially resolved transcriptomes—next

513 generation tools for tissue exploration. BioEssays 42, 1900221 (2020).

514 3. Ståhl, P. L. et al. Visualization and analysis of gene expression in tissue sections by 515 spatial transcriptomics. Science (80-. ). 353, 78-82 (2016).

5164 4e, B. et al. Integrating spatial gene expression and breast tumour morphology via deep 517 learning. Nat. Biomed. Eng. 1-8 (2020).

518 5. Berglund, E. et al. Spatial maps of prostate cancer transcriptomes reveal an unexplored 519 landscape of heterogeneity. Nat. Commun. 9, 1-13 (2018).

520 6. Thrane, K., Eriksson, H., Maaskola, J., Hansson, J. \& Lundeberg, J. Spatially resolved 521 transcriptomics enables dissection of genetic heterogeneity in stage III cutaneous 522 malignant melanoma. Cancer Res. 78, 5970-5979 (2018).

523 7. Maynard, K. R. et al. Transcriptome-scale spatial gene expression in the human 524 dorsolateral prefrontal cortex. Nat. Neurosci. 1-12 (2021).

525 8. Chen, W.-T. et al. Spatial transcriptomics and in situ sequencing to study Alzheimer's 526 disease. Cell 182, 976-991 (2020).

527 9. Van Dijk, D. et al. Recovering gene interactions from single-cell data using data diffusion. 
Cell 174, 716-729 (2018).

529

10. Jeong, H. \& Liu, Z. PRIME: a probabilistic imputation method to reduce dropout effects in single-cell RNA sequencing. Bioinformatics 36, 4021-4029 (2020).

531 11. Wagner, F., Yan, Y. \& Yanai, I. K-nearest neighbor smoothing for high-throughput singlecell RNA-Seq data. BioRxiv 217737 (2017).

533 12. Mongia, A., Sengupta, D. \& Majumdar, A. Mclmpute: matrix completion based imputation for single cell RNA-seq data. Front. Genet. 10, 9 (2019).

13. Huang, M. et al. SAVER: gene expression recovery for single-cell RNA sequencing. Nat. Methods 15, 539-542 (2018).

14. Linderman, G. C., Zhao, J. \& Kluger, Y. Zero-preserving imputation of scRNA-seq data using low-rank approximation. bioRxiv 397588 (2018).

15. Chen, Y.-T. et al. Serological analysis of Melan-A (MART-1), a melanocyte-specific protein homogeneously expressed in human melanomas. Proc. Natl. Acad. Sci. 93, 5915-5919 (1996).

16. Xie, S. et al. Expression of MCAM/MUC18 by human melanoma cells leads to increased tumor growth and metastasis. Cancer Res. 57, 2295-2303 (1997).

17. Zhou, Y. et al. Osteopontin expression correlates with melanoma invasion. J. Invest. Dermatol. 124, 1044-1052 (2005).

18. Shomali, N. et al. Heat shock proteins regulating toll-like receptors and the immune system could be a novel therapeutic target for melanoma. Curr. Mol. Med. 21, 15-24 (2021).

19. Hou, W., Ji, Z., Ji, H. \& Hicks, S. C. A systematic evaluation of single-cell RNAsequencing imputation methods. Genome Biol. 21, 1-30 (2020).

20. Mclnnes, L., Healy, J. \& Melville, J. Umap: Uniform manifold approximation and projection for dimension reduction. arXiv Prepr. arXiv1802.03426 (2018).

21. Langfelder, P. \& Horvath, S. WGCNA: an R package for weighted correlation network analysis. BMC Bioinformatics 9, 1-13 (2008).

22. Jones, A. R., Overly, C. C. \& Sunkin, S. M. The Allen brain atlas: 5 years and beyond.

557 23. Wold, S., Esbensen, K. \& Geladi, P. Principal component analysis. Chemom. Intell. Lab. 558 Syst. 2, 37-52 (1987).

559 24. Tarjan, R. Depth-first search and linear graph algorithms. SIAM J. Comput. 1, 146-160 560 (1972).

561 25. Virtanen, P. et al. \{SciPy\} 1.0: Fundamental Algorithms for Scientific Computing in 
$562 \quad$ Python. Nat. Methods 17, 261-272 (2020).

563 26. Pedregosa, F. et al. Scikit-learn: Machine Learning in \{P\}ython. J. Mach. Learn. Res. 12, 564 2825-2830 (2011).

565 27. Yu, G., Wang, L.-G., Han, Y. \& He, Q.-Y. clusterProfiler: an R package for comparing 566 biological themes among gene clusters. Omi. a J. Integr. Biol. 16, 284-287 (2012). 

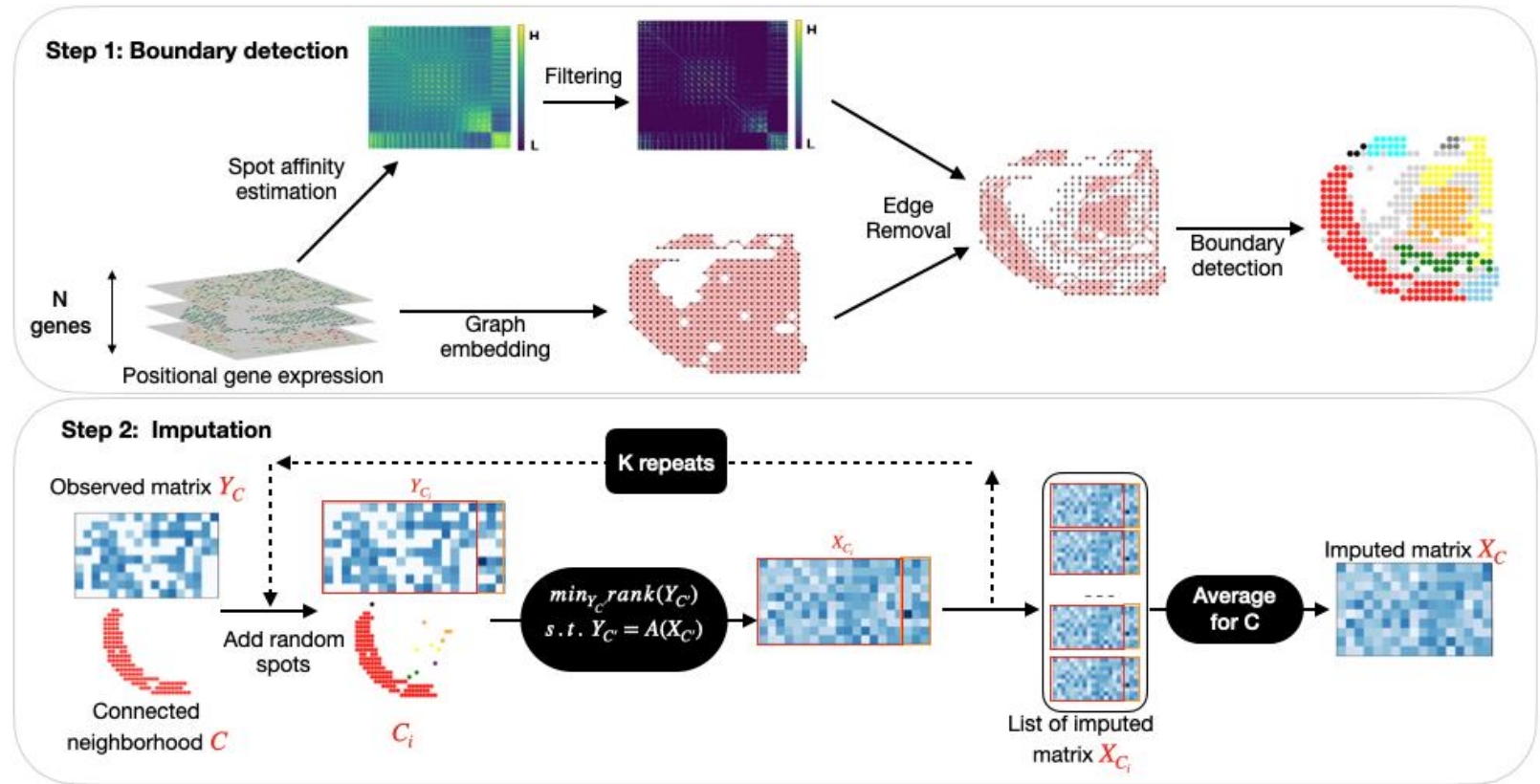

568 Figure 1. | The MIST pipeline. In step 1, region boundaries are detected by extracting locally 569 connected components through graph embedding and edge filtering. In step 2, missing values 570 for every detected region are estimated using the average of multiple runs of low-rank matrix 571 completion algorithm. 
a

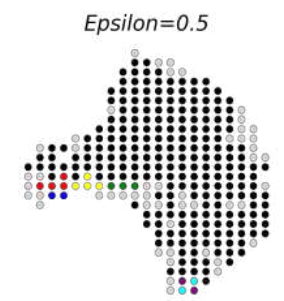

b

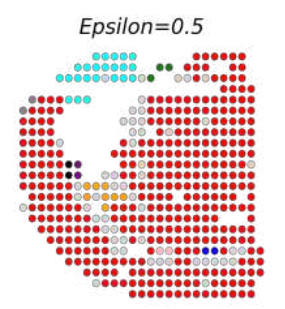

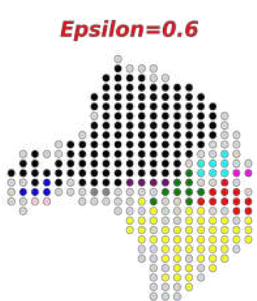

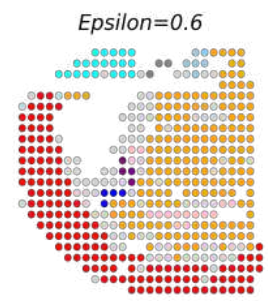

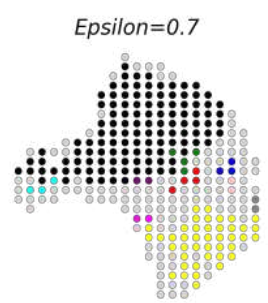

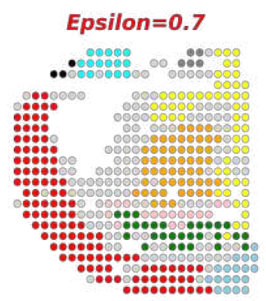

Manual annotation
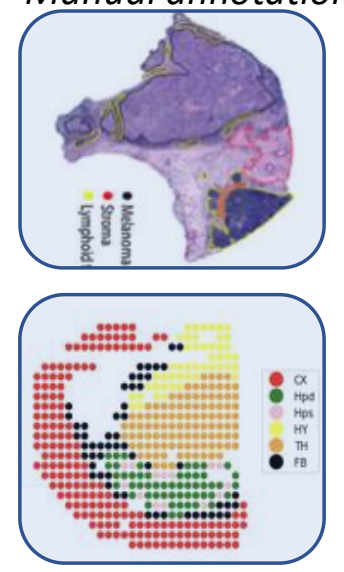

- NS $\log _{2} \mathrm{FC} \odot \mathrm{p}$-value $\mathrm{p}$-value and $\log _{2} \mathrm{FC}$

C
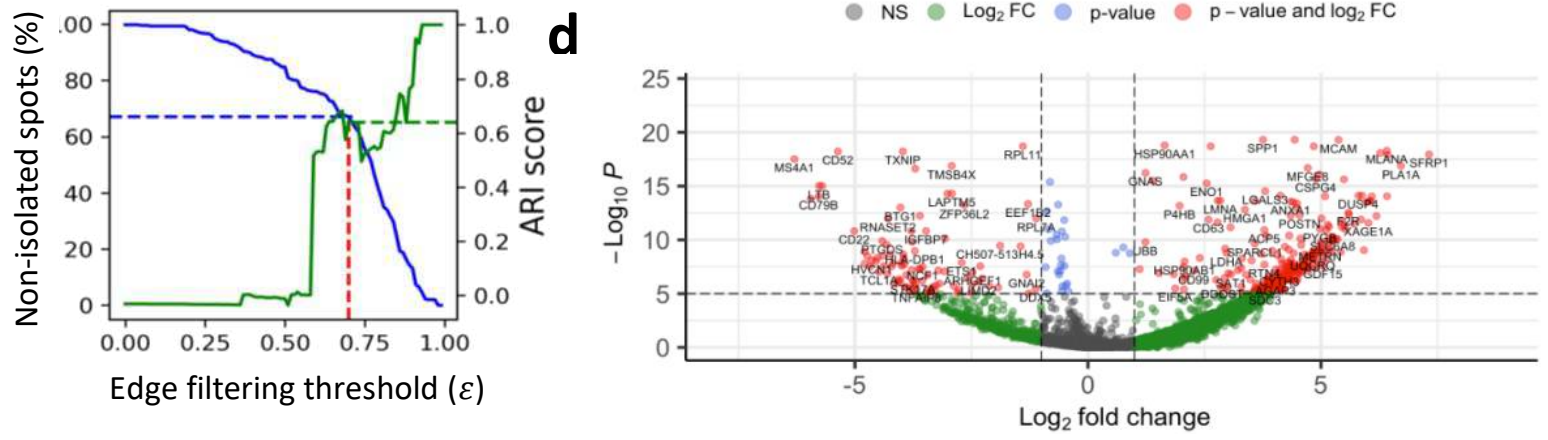

e
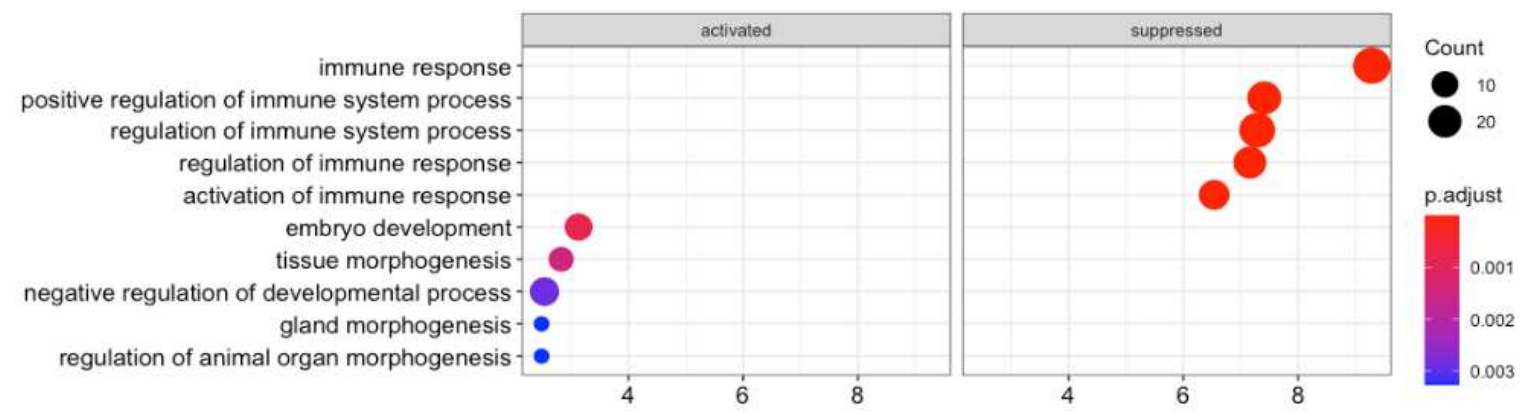

Figure 2. | MIST detected regions agree with manual annotations. a, Melanoma tissue regions detected using different epsilon values. Column 2 with epsilon of 0.6 was selected by MIST. Column 4 is the pathological annotation on the aligned histological staining image. b, Mouse brain tissue regions detected using different epsilon values. Column 3 with epsilon of 0.7 was selected by MIST. Column 4 is the spot-level manual annotation from the original study. c, Percentage of non-isolated spots (left y-axis, blue curve) and adjusted rand index (ARI, right yaxis, green curve) as functions of edge-filtering parameter epsilon. $\mathbf{d}$, Volcano plot of differential expressed genes in tumor region contrasted to lymphoid region of the Melanoma sample. e, Gene ontologies activated and suppressed for MIST-detected melanoma tumor region compared relatively to lymphoid region. 


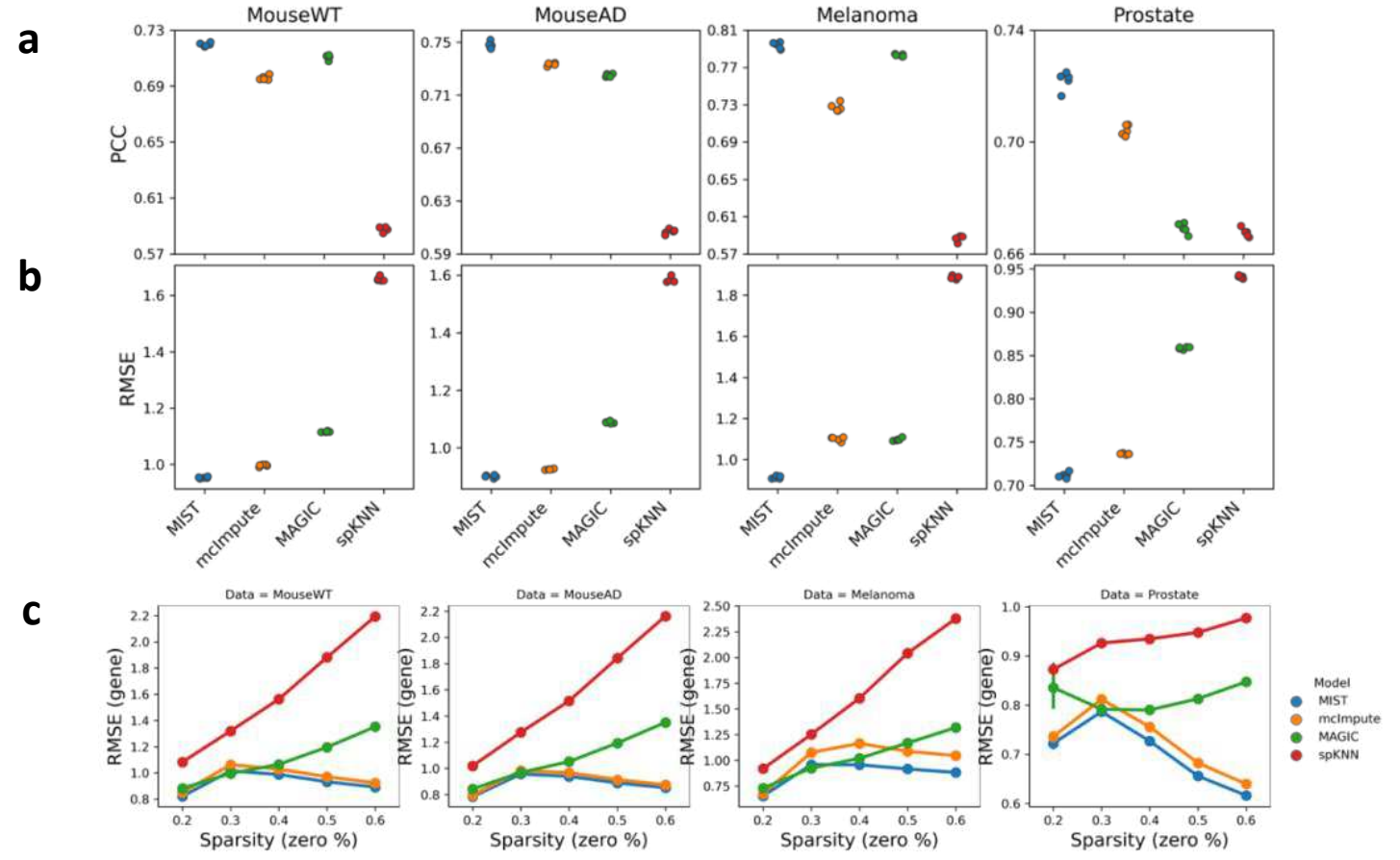

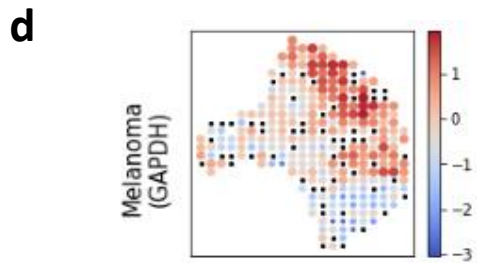

Holdout

e

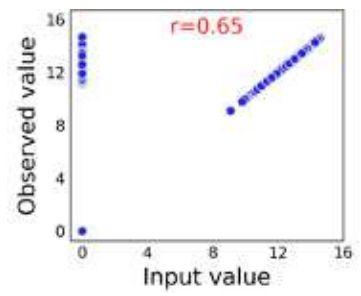

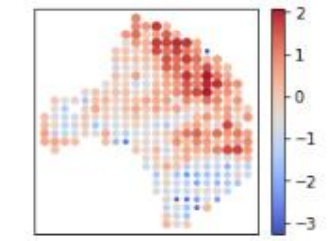

Denoised

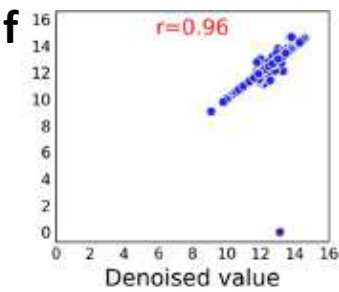

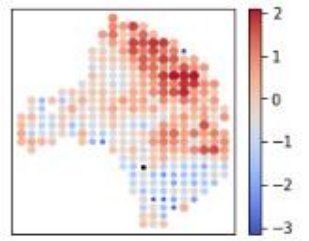

Observed

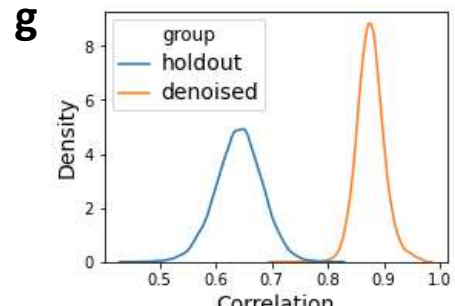

\section{Figure 3. | MIST's outperforms other imputation methods in holdout experiments. $a-b$,} Holdout experiment performance across multiple data sets using metrics $\mathbf{a}$, PCC and $\mathbf{b}, \mathrm{RMSE}$. Each column is an individual data set. Points in the same color indicate the 5 non-overlap fold of tests for each model. c, Gene-level performance of each model represented by the RMSE ( $y$ axis) as a function of zero-value percentage of the gene expression values (sparsity, $x$-axis). d, Expression patterns recovered for gene GAPDH in the Melanoma sample. From left to right shows the spatial pattern of the input to MIST with holdouts, the denoised gene expression pattern and the original observed gene expression pattern. Color and size indicate relative gene expression abundance. e, the correlation between holdout input and observed GAPDH expression values. $\mathbf{f}$, the correlation between MIST denoised and observed GAPDH expression values. $\mathbf{g}$, distribution of the gene-level correlation between original non-zero gene expression values and the holdout-input (blue), and denoised values (orange), respectively. 
a
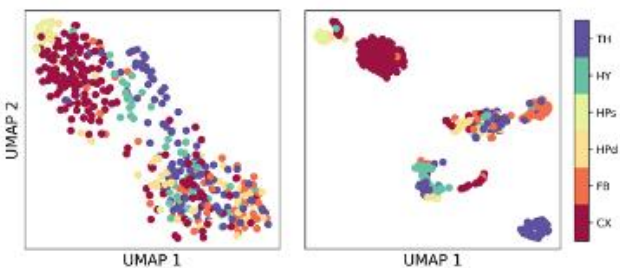

b
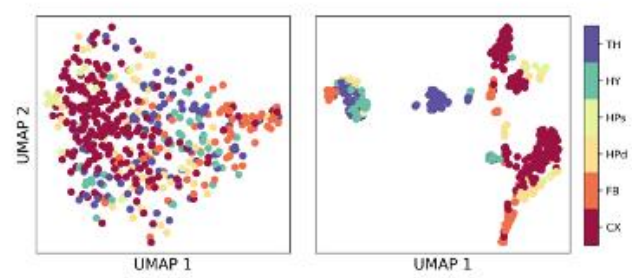

C
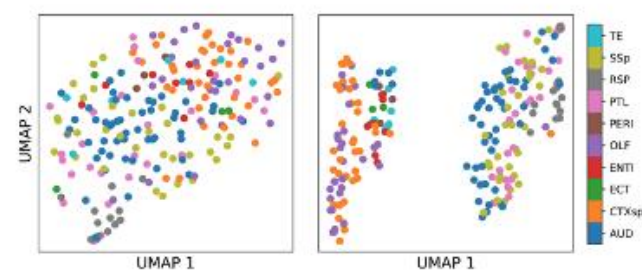
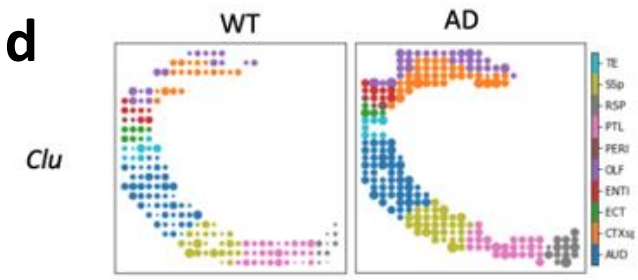

e

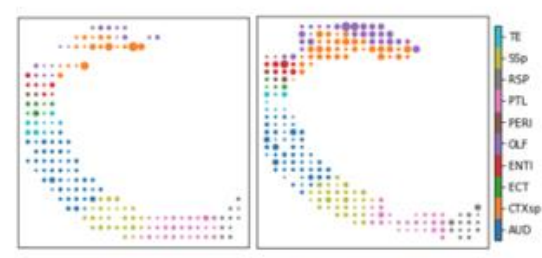

f
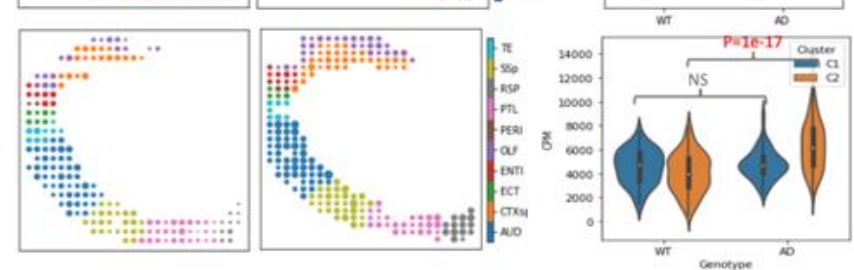

597

598

599

600

601

602

603

604

605

606

607

608

609
Figure 4. I MIST identified intra-cortex heterogeneity within Alzheimer's Disease (AD) mouse brain. a, UMAP of Mouse WT brain using original observed ST data (left) and MIST-denoised ST data (right). b, UMAP of Mouse AD brain using original observed ST data (left) and MISTdenoised ST data (right). c, UMAP of Mouse AD cortex region using original observed ST data (left) and MIST-denoised ST data (right). d-f, Examples of AD up-regulated genes that are activated in both clusters (d), only significantly upregulated in Cluster 1 (e, CTXsp: cortical subplate, OLF: olfactory, ENTI: entorhinal, TE: temporal association, ECT: ectorhinal, and PERI: perirhinal areas) and Cluster 2 (f, AUD: auditory, PTL: posterior parietal association area, RSP: retrosplenial area, SSp: primary somatosensory). Left, spatial expression pattern in WT mouse brain cortex; middle, spatial expression pattern in AD mouse brain cortex; right, boxplot of CPM grouped by genotype and spatial clusters. 
a
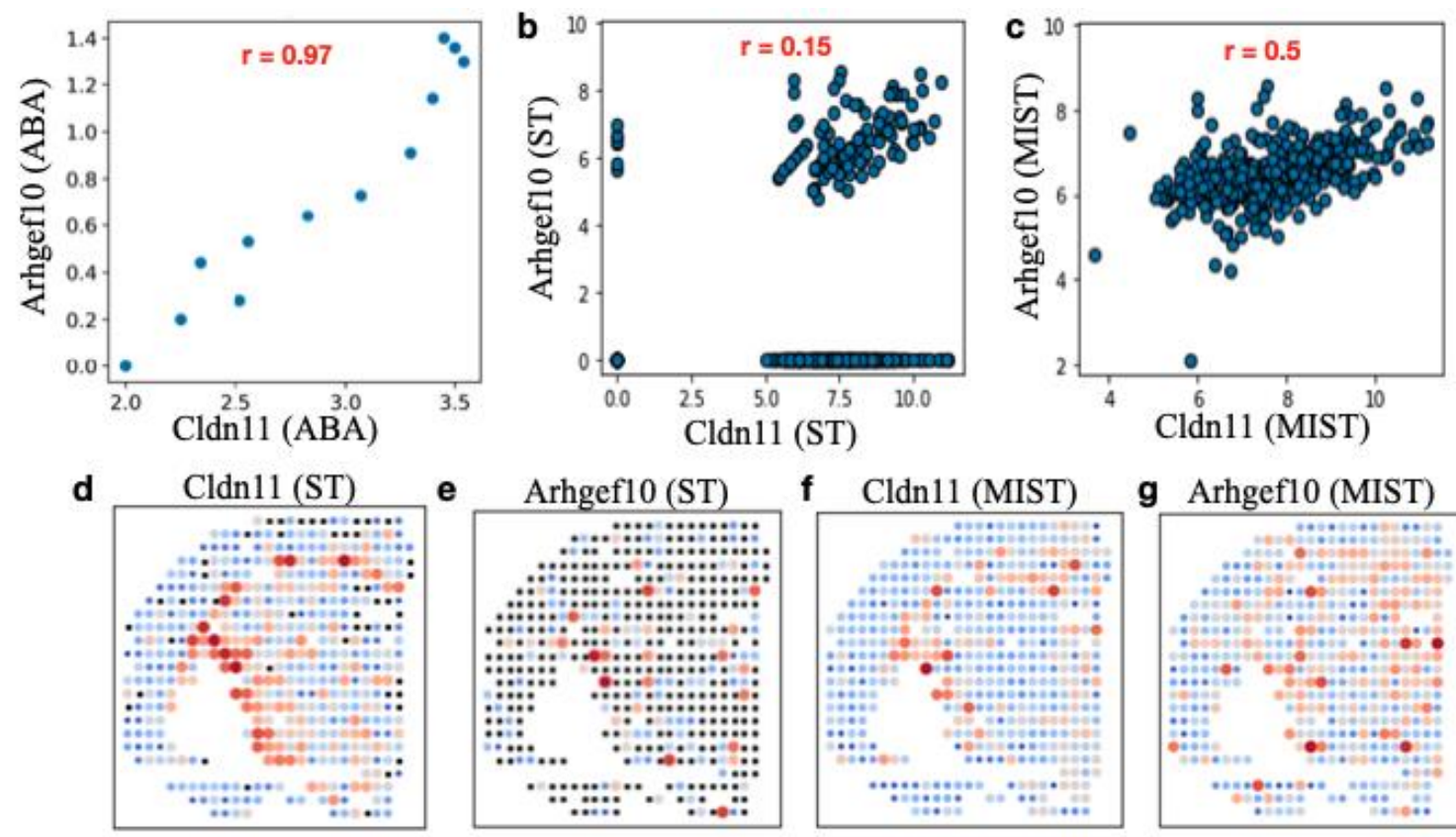

g Arhgef10 (MIST)

h

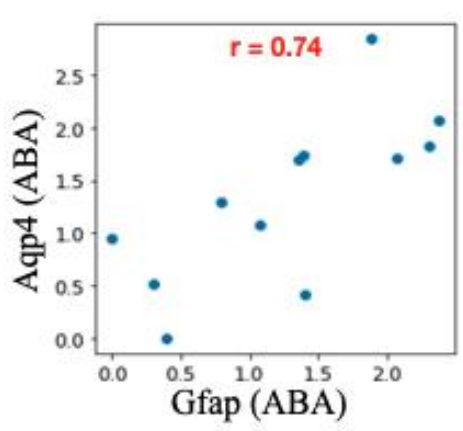

i

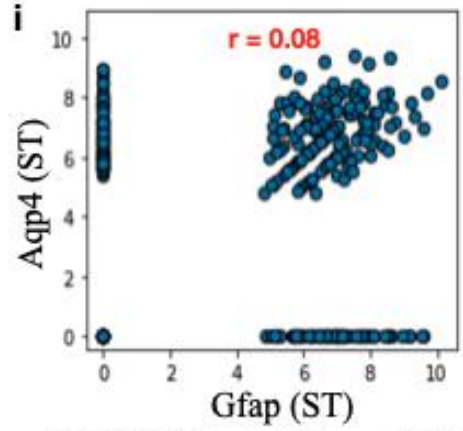

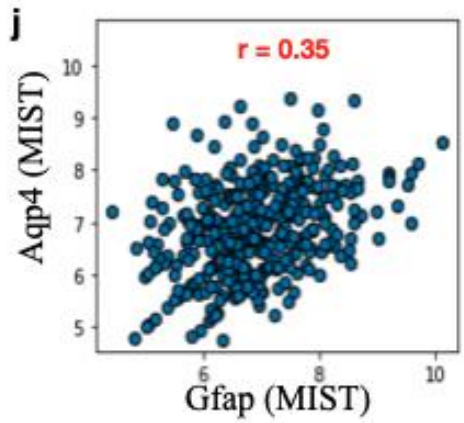

$\mathbf{k}$ I
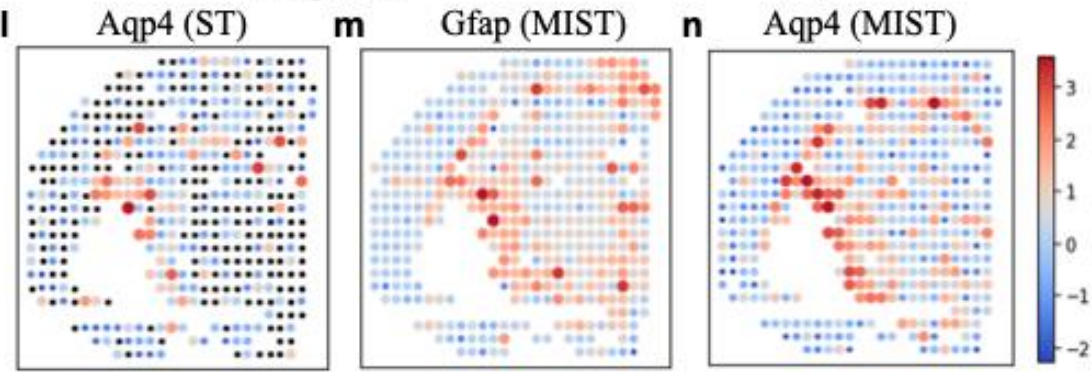

Figure 5. I MIST recovers spatially co-expressed gene pairs. a-c, spatial correlation of Cldn11Arhgef10 in the (a) Allen Brain Atlas reference, (b) original ST data and (c) MIST-denoised ST data. $\mathbf{d}$, expression patterns of gene Cldn11 using the original ST data. e, expression patterns of gene Arhgef10 using the original ST data. f, expression patterns of gene Cldn11 using the MISTdenoised data. $\mathbf{g}$, expression patterns of gene Arhgef10 using the MIST-denoised data. $\mathbf{h}-\mathbf{j}$, spatial correlation of Gfap-Aqp4 in the (h) Allen Brain Atlas reference, (i) original ST data and (j) MIST-denoised ST data. $\mathbf{k}$, expression patterns of gene Gfap using the original ST data. I, expression patterns of gene Apq4 using the original ST data. $\mathbf{m}$, expression patterns of gene Gfap using the MIST-denoised data. $\mathbf{n}$, expression patterns of gene Aqp4 using the MISTdenoised data. 


\section{Supplementary Files}

This is a list of supplementary files associated with this preprint. Click to download.

- extendedfigures.docx 